\title{
MED12 exon 2 mutations are common in uterine leiomyomas from South African patients
}

\author{
Netta Mäkinen ${ }^{1}$, Hanna-Riikka Heinonen ${ }^{1}$, Shane Moore ${ }^{2}$, Ian P.M. Tomlinson ${ }^{3}$, \\ Zephne M. van der Spuy ${ }^{2}$, and Lauri A. Aaltonen ${ }^{1}$ \\ ${ }^{1}$ Department of Medical Genetics, Genome-Scale Biology Research Program, University of Helsinki, Helsinki, Finland \\ 2 Department of Obstetrics and Gynaecology, Faculty of Health Sciences, University of Cape Town, South Africa \\ ${ }^{3}$ Wellcome Trust Centre for Human Genetics and NIHR Comprehensive Biomedical Research Centre, Nuffield Department of \\ Clinical Medicine, Roosevelt Drive, University of Oxford, Oxford OX3 7BN, UK
}

Correspondence to: Lauri A. Aaltonen, email: lauri.aaltonen@helsinki.fi

Keywords: Uterine leiomyoma, fibroid, MED12, ethnicity

Received: November 30, 2011, Accepted: December 9, 2011, Published: December 19, 2011

Copyright: ( ) Mäkinen et al. This is an open-access article distributed under the terms of the Creative Commons Attribution License, which permits unrestricted use, distribution, and reproduction in any medium, provided the original author and source are credited.

ABSTRACT:

\begin{abstract}
Uterine leiomyomas, or fibroids, are extremely common tumors. Regardless of their benign nature, fibroids can cause considerable morbidity. Women with African ancestry have a threefold increased risk of developing uterine leiomyomas with a greater symptom severity when compared to white women. Recently, we demonstrated that exon 2 of the MED12 gene is somatically altered in up to 70 per cent of uterine leiomyomas in a series of Finnish (Caucasian) patients. To validate these results in other populations, we sequenced a set of $\mathbf{2 8}$ uterine leiomyomas for MED12 exon 2 mutations from 18 different Black African or Coloured South African patients. We observed 14 mutation positive lesions (50\%). When corrected by tumor size, these results are very similar to those derived in the Finnish material. This study confirms a major role of MED12 in the genesis of leiomyomas, regardless of ethnicity.
\end{abstract}

\section{INTRODUCTION}

Uterine leiomyomas, also known as fibroids, are benign tumors for which the lifetime risk in women over the age of 45 has been estimated to exceed $60 \%$ [1]. Fibroids arise from the smooth muscle cells of the myometrium and can cause significant morbidity, such as abnormal uterine bleeding, abdominal pain and discomfort, pregnancy complications and even infertility [2]. Fibroids are the most common cause for hysterectomy, and they have a considerable socio-economic impact [34].

Uterine leiomyomas are monoclonal, oestrogen and progesterone dependent tumors, which occur in women of reproductive age, and typically regress with the onset of menopause. On the other hand, parity and use of oral contraceptives have been suggested to protect women from the development of fibroids [1]. It is also known that uterine leiomyomas do not affect all ethnicities equally. Women with African ancestry have a threefold risk of developing uterine leiomyomas compared with white women [5], and Africans have also been reported to have an earlier age at onset with larger, more numerous, and more rapidly-growing fibroids [6-7]. In addition to ethnicity, other factors, such as family history, smoking, alcohol intake, hypertension and increased body weight have been proposed to increase the risk of developing uterine leiomyomas $[6,8-10]$.

We recently identified various somatic mutations in exon 2 of the mediator complex subunit 12 (MED12) gene, in as many as $70 \%$ of the studied uterine leiomyomas obtained from patients of Finnish (Caucasian) origin [11]. The mutation hot spot affected an evolutionary conserved region of the MED12 protein, and according to our results, large fibroids tended to have slightly fewer mutations than small fibroids. MED12 is part of the 26-subunit Mediator complex which is thought to regulate global as well as gene-specific transcription by bridging distant regulatory DNA elements to the RNA polymerase II initiation complex [12].

The aim of this study was to investigate the frequency of MED12 exon 2 mutations in uterine leiomyomas of South African patients to confirm that MED12 has a major role in the genesis of this tumor type in populations 
other than Finns. Thus, we screened a total of 28 uterine fibroids from 18 individual patients for these mutations.

\section{RESULTS}

We sequenced a set of 28 uterine leiomyomas from 18 South African patients. Fourteen leiomyomas (50\%) harbored a mutation in MED12 exon 2 (Table 1). Eight of these mutations were located in codon 44 . In addition, two fibroids (7\%) displayed a missense mutation in codon 36 and one fibroid $(3.6 \%)$ in codon 43 . We also observed two (7\%) exonic insertion-deletion type mutations and one somatic intronic $\mathrm{T}$ to A mutation (3.6\%) eight base pairs upstream of the splice acceptor site of exon 2. All three mutations are predicted to result in an in-frame transcript. The somatic nature of the mutations was verified in all cases where normal tissue DNA was available (nine). Nine patients did not have any mutations in MED12 exon 2.

The difference between the frequency of mutation positive fibroids in women with mixed ancestry (Coloured) and Black South African women was statistically significant when compared with the frequency of mutation positive lesions in Finnish (Caucasian) women (p-value $=0.045)[11]$. However, if corrected by tumor size, the results in the two series were very similar $(p$-value $=0.69)$.

\section{DISCUSSION}

To our knowledge, this is the first description of $M E D 12$ exon 2 mutation analysis in uterine leiomyomas from other than white women. In this study, a set of 28 uterine leiomyomas from 18 South African patients was sequenced for MED12 exon 2 mutations to study the role of MED12 in tumorigenesis of fibroids also in other populations than Finns. Altogether, 14 (50\%) mutation positive lesions were observed.

Ethnicity is an important epidemiological risk factor for uterine leiomyomas in the general population. The effect of race on incidence and severity of fibroids is particularly significant. Several studies have reported a higher incidence of fibroids among Black women than other racial and ethnic groups including Caucasian, Hispanic, and Asian women [6, 10, 13]. Moreover, Black women tend to have more severe disease than Caucasian women, including an earlier age at diagnosis and at hysterectomy, with larger, more numerous, and more rapidly growing fibroids [6-7, 13].

The reasons for ethnic variation in uterine leiomyoma

Table 1: Patient information and $M E D 12$ exon 2 mutation status of the studied fibroids.

\begin{tabular}{|c|c|c|c|c|c|c|c|}
\hline Patient & $\begin{array}{c}\text { Age at } \\
\text { Diagnosis }\end{array}$ & Ethnicity & $\begin{array}{c}\text { Number of } \\
\text { Fbroids }\end{array}$ & Fbroid & Size & Status of MED12 exon 2 & Myometrium \\
\hline \multirow[t]{2}{*}{ FG106 } & 50 & Black South African & 3 & FG106_1 & Fundal $20 \times 10 \mathrm{~cm}$ & c. $130 G>A, p . G 44 S$ & $x$ \\
\hline & & & & FG106_2 & Anterior $15 \times 15 \mathrm{~cm}$ & c.100-8T>A, p.E33_D34insPQ & \\
\hline FG107 & 48 & Coloured & Multiple & FG107_1 & Posterior $3.7 \times 4.0 \mathrm{~cm}$ & c.107T>G, p.L36R & $x$ \\
\hline \multirow[t]{3}{*}{ FG108 } & 41 & Coloured & Not reported & FG108_1 & $4.4 \times 4.2 \mathrm{~cm}$ & $w t$ & $x$ \\
\hline & & & & FG108_2 & $2.5 \times 3.3 \mathrm{~cm}$ & c. $131 \mathrm{G}>\mathrm{A}, \mathrm{p} . \mathrm{G} 44 \mathrm{D}$ & \\
\hline & & & & FG108_3 & Not reported & c.149_163del15, p.A50_D54del & \\
\hline \multirow[t]{2}{*}{ FG109 } & 46 & Coloured & Multiple & FG109_1 & Not reported & $w t$ & $x$ \\
\hline & & & & FG109_2 & Not reported & $w t$ & \\
\hline \multirow[t]{2}{*}{ FG141 } & 27 & Black South African & 15 & FG141_1 & Fundal no size & c. $130 G>C$, p.G44R & \\
\hline & & & & FG141_3 & Lateral no size & c. $131 \mathrm{G}>\mathrm{A}$, p.G44D & \\
\hline FG142 & 59 & Black South African & Multiple & FG142_2 & Lateral no size & c. $107 T>$ G, p.L36R & $x$ \\
\hline FG146 & 39 & Coloured & 1 & FG146_1 & $7.0 \times 7.1 \mathrm{~cm}$ & $w t$ & $x$ \\
\hline \multirow[t]{2}{*}{ FG147 } & 30 & Coloured & 2 & FG147_1 & Anterior $6.7 \times 6.7 \mathrm{~cm}$ & wt & \\
\hline & & & & FG147_2 & Posterior no size & c.130G>T, p.G44C & \\
\hline FG149 & 48 & Coloured & 2 & FG149_1 & $1.3 \times 1.3 \mathrm{~cm}$ & $w t$ & $x$ \\
\hline \multirow[t]{2}{*}{ FG150 } & 47 & Coloured & 2 & FG150_1 & Anterior $4.3 \times 4.1 \mathrm{~cm}$ & $w t$ & $x$ \\
\hline & & & & FG150_2 & Posterior $4.5 \times 5.0 \mathrm{~cm}$ & $w t$ & \\
\hline \multirow[t]{2}{*}{ FG151 } & 39 & Coloured & 2 & FG151_1 & Anterior no size & $w t$ & \\
\hline & & & & FG151_2 & Posterior no size & $w t$ & \\
\hline \multirow[t]{2}{*}{ FG152 } & 41 & Coloured & 2 & FG152_1 & Anterior $7.3 \times 5.9 \mathrm{~cm}$ & c. $131 \mathrm{G}>\mathrm{C}, \mathrm{p} . \mathrm{G} 44 \mathrm{~A}$ & $x$ \\
\hline & & & & FG152_2 & Posterior $11.0 \times 7.5 \mathrm{~cm}$ & c. $131 \mathrm{G}>\mathrm{A}, \mathrm{p} . \mathrm{G} 44 \mathrm{D}$ & \\
\hline FG153 & 50 & Coloured & 2 & FG153_1 & Anterior no size & c. $128 \mathrm{~A}>$ C, p. $Q 43 P$ & \\
\hline FG154 & 45 & Black South African & 1 & FG154_1 & Inferior $5.0 \times 4.5 \mathrm{~cm}$ & $w t$ & $x$ \\
\hline \multirow[t]{2}{*}{ FG155 } & 33 & Coloured & Multiple & FG155_1 & Lateral no size & c.122_148del27, p.V41_P49 & $x$ \\
\hline & & & & FG155_2 & Lateral no size & c. $131 G>A, p . G 44 D$ & \\
\hline FG157 & 32 & Black South African & Multiple & FG157_1 & Not reported & $w t$ & $x$ \\
\hline FG166 & 48 & Coloured & Multiple & FG166_1 & Anterior no size & wt & $x$ \\
\hline FG169 & 41 & Black South African & 2 & FG169_1 & Posterior $17.8 \times 11.5 \mathrm{~cm}$ & $w t$ & $x$ \\
\hline
\end{tabular}


occurrence are unknown. Various possible causes for higher prevalence and greater symptom severity among Black women have been proposed. For example, the differences may be due to genes that confer increased risk for poor outcome. Uterine leiomyomas are hormonally responsive tumors, and for instance, Black women have been reported to have a higher prevalence of oestrogen receptor- $\alpha$ PP variant than white women [14]. The variant has been associated with an increased risk of uterine leiomyomas in both ethnicities.

Our studies demonstrate that fibroids from both Caucasians as well as women with African descent frequently harbor mutations in MED12 exon 2. The South African series displayed significantly fewer mutationpositive lesions than the previously published Finnish series. However, the tumors in the South African series tended to be larger, and because the results in the Finnish series had indicated an inverse correlation between MED12 mutations and tumor size, we analyzed the results after correction of this tumor characteristic. Indeed, after this correction the results were very similar. While further work remains to be done to clarify the reasons behind the observed differences, this study confirms a major role of MED12 in the tumorigenesis of leiomyomas, regardless of ethnicity.

\section{MATERIALS AND METHODS}

\section{Patient Material}

DNA of 28 uterine leiomyoma and 14 myometrium samples was obtained from the Department of Obstetrics and Gynaecology, Faculty of Health Sciences, University of Cape Town, South Africa. DNA had been extracted from fresh frozen tissue samples. Altogether 18 individual patients were included to this study and from each patient 1-3 uterine leiomyomas were examined (Table 1). The patient series comprised of twelve women with mixed ancestry (Coloured) and six Black South African women. This study was approved by the local Human Research Ethics Committee (REF: 008/1995 and REF: 433/2011).

\section{Amplification of MED12 exon 2}

Using previously reported primer sequences [11] the desired DNA fragment was amplified with AmpliTaqGold $\AA$ enzyme (Applied Biosystems, Foster City, CA, USA). The PCR products were purified using ExoSAP-IT PCR Purification Kit (USB Corporation, Cleveland, $\mathrm{OH}, \mathrm{USA}$ ) and the sequencing reactions were performed utilizing the Big Dye Terminator v.3.1 Kit (Applied Biosystems, Foster City, CA, USA) according to the manufacturer's instructions. Sequencing was performed on an ABI3730 Automatic DNA Sequencer
(Applied Biosystems at FIMM Genome and Technology Centre Finland). The sequence graphs were analyzed both manually and on computer with Mutation Surveyor program (Softgenetics, State College, PA, USA).

\section{Statistical Analysis}

Statistical analyses were performed using R software, version 2.14.0. Differences between the proportion of mutation positive lesions in uterine leiomyomas with Finnish and South African patients were undertaken with Pearson's $\chi^{2}$ test with $1 d f$. Also the differences between the frequency of large (at least $5.5 \mathrm{~cm}$ diameter) mutation positive lesions in the South African series versus the Finnish series, was evaluated with Fisher's exact test.

\section{ACKNOWLEDGEMENTS}

We would like to thank S. Nieminen for technical assistance. This study was supported by the Academy of Finland (Center of Excellence in Translational GenomeScale Biology grant 6302352, and grants 1124270, 212901, and 214323), the Sigrid Jusélius Foundation, the Cancer Society of Finland, and the National Research Foundation of SA (NRF).

\section{REFERENCES}

1. Okolo S. Incidence, aetiology and epidemiology of uterine fibroids. Best Practice \& Research Clinical Obstetrics \& Gynaecology. 2008; 22: 571-88.

2. Stewart EA. Uterine fibroids. Lancet. 2001; 357: 293-98.

3. Flynn M, Jamison M, Datta S, Myers E. Health care resource use for uterine fibroid tumors in the United States. American Journal of Obstetrics and Gynecology. 2006;195: 955-64.

4. Hartmann KE, Birnbaum H, Ben-Hamadi R, Wu EQ, Farrell MH, Spalding J, Stang P. Annual costs associated with diagnosis of uterine leiomyomata. Obstetrics and Gynecology. 2006; 108: 930-37.

5. Day Baird D, Dunson DB, Hill MC, Cousins D, Schectman JM. High cumulative incidence of uterine leiomyoma in black and white women: ultrasound evidence. American Journal of Obstetrics and Gynecology. 2003; 188: 100-7.

6. Marshall LM, Spiegelman D, Barbieri RL, Goldman MB, Manson JE, Colditz GA, Willett WC, Hunter DJ. Variation in the incidence of uterine leiomyoma among premenopausal women by age and race. Obstetrics and Gynecology. 1997; 90: 967-73.

7. Huyck KL, Panhuysen CI, Cuenco KT, Zhang J, Goldhammer H, Jones ES, Somasundaram P, Lynch AM, Harlow BL, Lee H, Stewart EA, Morton CC. The impact of race as a risk factor for symptom severity and age at diagnosis of uterine leiomyomata among affected sisters. 
American Journal of Obstetrics and Gynecology. 2008; 198; 168.e1-e9.

8. Wise LA, Palmer JR, Harlow BL, Spiegelman D, Stewart EA, Adams-Campbell LL, Rosenberg L. Risk of uterine leiomyomata in relation to tobacco, alcohol and caffeine consumption in the Black Women's Health Study. Human Reproduction (Oxford, England). 2004; 19: 1746-54.

9. Wise LA, Palmer JR, Spiegelman D, Harlow BL, Stewart EA, Adams-Campbell LL, Rosenberg L. Influence of body size and body fat distribution on risk of uterine leiomyomata in U.S. black women. Epidemiology (Cambridge, Mass.). 2005; 16: 346-54.

10. Templeman C, Marshall SF, Clarke CA, Henderson KD, Largent J, Neuhausen S, Reynolds P, Ursin G, Bernstein L. Risk factors for surgically removed fibroids in a large cohort of teachers. Fertility and Sterility. 2009; 92: 143646.

11. Mäkinen N, Mehine M, Tolvanen J, Kaasinen E, Li Y, Lehtonen HJ, Gentile M, Yan J, Enge M, Taipale M, Aavikko M, Katainen R, Virolainen E, Böhling T, Koski TA, Launonen V, Sjöberg J, Taipale J, Vahteristo P, Aaltonen LA. MED12, the mediator complex subunit 12 gene, is mutated at high frequency in uterine leiomyomas. Science. 2011; 334: 252-55.

12. Taatjes DJ. The human Mediator complex: a versatile, genome-wide regulator of transcription. Trends in Biochemical Sciences. 2010; 35: 315-22.

13. Kjerulff KH, Langenberg $\mathrm{P}$, Seidman JD, Stolley PD, Guzinski GM. Uterine leiomyomas. Racial differences in severity, symptoms and age at diagnosis. The Journal of Reproductive Medicine. 1996; 41: 483-90.

14. Al-Hendy A, Salama SA. Catechol-O-methyltransferase polymorphism is associated with increased uterine leiomyoma risk in different ethnic groups. Journal of the Society for Gynecologic Investigation. 2006; 13: 136-44. 